\title{
Primary Health Care System: A Framework for Strengthen- ing and Sustaining COVID-19 Outbreak Response in Nigeria
}

\author{
Short Communication
}

Volume 3 Issue 1- 2022

\begin{abstract}
Author Details
Elvis Efe Isere*

Department of Epidemiology and Medical Statistics, University of Ibadan, Nigeria

*Corresponding author

Elvis Efe Isere, Department of Epidemiology and Medical Statistics, University of Ibadan, Ibadan, Nigeria
\end{abstract}

Article History

Received: January 21, 2022 Accepted: January 21, 2022 Published: January 27, 2022

\begin{abstract}
The COVID-19 pandemic has been ongoing for about 2 years and is currently creating a deadly ripple effect on the health of people in Nigeria and across the world, overwhelming healthcare infrastructures and the unprepared health system capacity of several countries thereby threatening the significant gains in health and human progress and development made over several decades. Addressing this ongoing global health "monster" called COVID-19 and providing people with essential services while responding to and recovering from the pandemic in Nigeria demands a strong and resilient health system, especially at the primary health care (PHC) level, that can address people's diverse health needs at every stage of life. This paper discusses the need to strengthen the capacity of the PHC system especially during the COVID-19 outbreak response and identify key roles the PHC system could play in sustaining and winning the war against COVID-19 in Nigeria.
\end{abstract}

Keywords: Primary health care; COVID-19 pandemic; Outbreak response; Nigeria

\begin{abstract}
Abbreviations: Ag-RDT: Antigen Rapid Diagnostic Test; DSNOs: Disease Surveillance and Notification Officers; COVID-19: Coronavirus Disease 2019; FCT: Federal Capital Territory; FMoH: Federal Ministry of Health; LGA: Local Government Areas, NCDC: Nigeria Center for Disease Control; PHCC: Primary Health Center Coordinator; PHC: Primary Health Care; PHCs: Primary Health Centers; PHCPI: Primary Health Care Performance Initiative; PHEOCs: Public Health Emergency Operation Centers; RRT: Rapid Response Team; WHO: World Health Organization
\end{abstract}

\section{Introduction}

As several countries struggle with containing the COVID-19 pandemic, the need for strong primary health care cannot be overemphasized [1]. PHC is people's first point of contact with the health system [1]. It's where they can turn for essential services like maternal \& child health care, treatment for chronic diseases and many more. It has also become the first line of defense for COVID-19 [1]. The PHC framework is the most inclusive, comprehensive, effective and efficient means to protect people's health and wellbeing especially during public health crises such as COVID-19 as it is the closest health care delivery system to the people [2]. The PHC framework is capable of providing $80-90 \%$ of people's health needs across their lifetime [3].

As COVID-19 continues to overwhelm the Nigerian health systems, PHC can play a key part in the outbreak preparedness, response and can also serve as the foundation for recovery [1]. In Nigeria over the years, the PHC system has occupied the front line of a country's response against several infectious outbreaks from Lassa fever, cholera, Measles, yellow fever and other public health crises like COVID-19 [4]. It has played very important roles in effectively and equitably stopping disease spread through prompt detection, reporting and tracking (surveillance), diagnosis, and conducting vaccination campaigns (routine and mass vaccination exercises) while responding to communities' comprehensive health demands $[1,4]$.

PHC system is the bedrock of the country's health system and forms the foundation of effective health systems capable of delivering quality essential and routine health care services to avoid further preventable illness and death both during and beyond the pandemic [1,5]. In order to strengthen and sustain the COVID-19 outbreak response in Nigeria, a comprehensive and participatory approach is required [4]. The PHC system is considered to be a more appropriate approach 
to health, and the health system, improving access to health services, as well as disease prevention and control [6]. This paper discussed the current COVID-19 outbreak situation, an overview of the PHC system in Nigeria and explains key roles the PHC system could play to strengthen and sustain the ongoing outbreak response against the COVID-19 outbreak in Nigeria.

\section{Overview of COVID-19 Outbreak and Response Strategies in Nigeria}

Nigeria is the most populous country in Africa, with an estimated population of over 160 million [7] and a growth rate of $3.8 \%$ per annum. Nigeria has six regional zones with varying ecologies, climates and population characteristics. The zones are divided into 36 states and the federal capital territory, which is further divided into 774 LGAs or districts and 8812 administrative wards [8].

The country is currently one of the nations in the African region that is most affected by the COVID-19 pandemic. With the outbreak of COVID-19 still very active in Nigeria, the country currently ranks top among other countries within the African region with ongoing transmission of COVID-19 disease [9,10]. Since the report of the index case in Nigeria in February 2020 by the Nigeria Center for Disease
Control (NCDC), daily records of confirmed cases have been reported in all states in the country $[9,10]$. As of 21st December 2021, Nigeria has recorded a total of 225, 255 confirmed cases and 2985 deaths in all 36 states and Federal Capital Territory. The country has experienced three waves of the dreadful COVID-19 outbreak and presently has entered a fourth wave of the outbreak with the threat of the recent omicron variant of the virus [9].

Since the inception of the COVID-19 outbreak in Nigeria, the Nigerian government through Nigeria Center for Disease Control (NCDC) has implemented several outbreak response strategies ranging from enhanced surveillance system for suspected case detection and reporting (active surveillance, active case search, contact tracing), strengthening public health laboratory capacity for testing suspected cases, the establishment of treatment centers including training of medical professionals for quality management of confirmed cases, development and the dissemination of public health advisory on preventive measures against COVID-19 through several risk communication channels, to strengthening the coordination of human, financial and material resources for the outbreak control through the establishment of public health emergency operation centers (PHEOCs) across the several states for daily coordination of the outbreak response activities $[9,11]$ Figure 1.

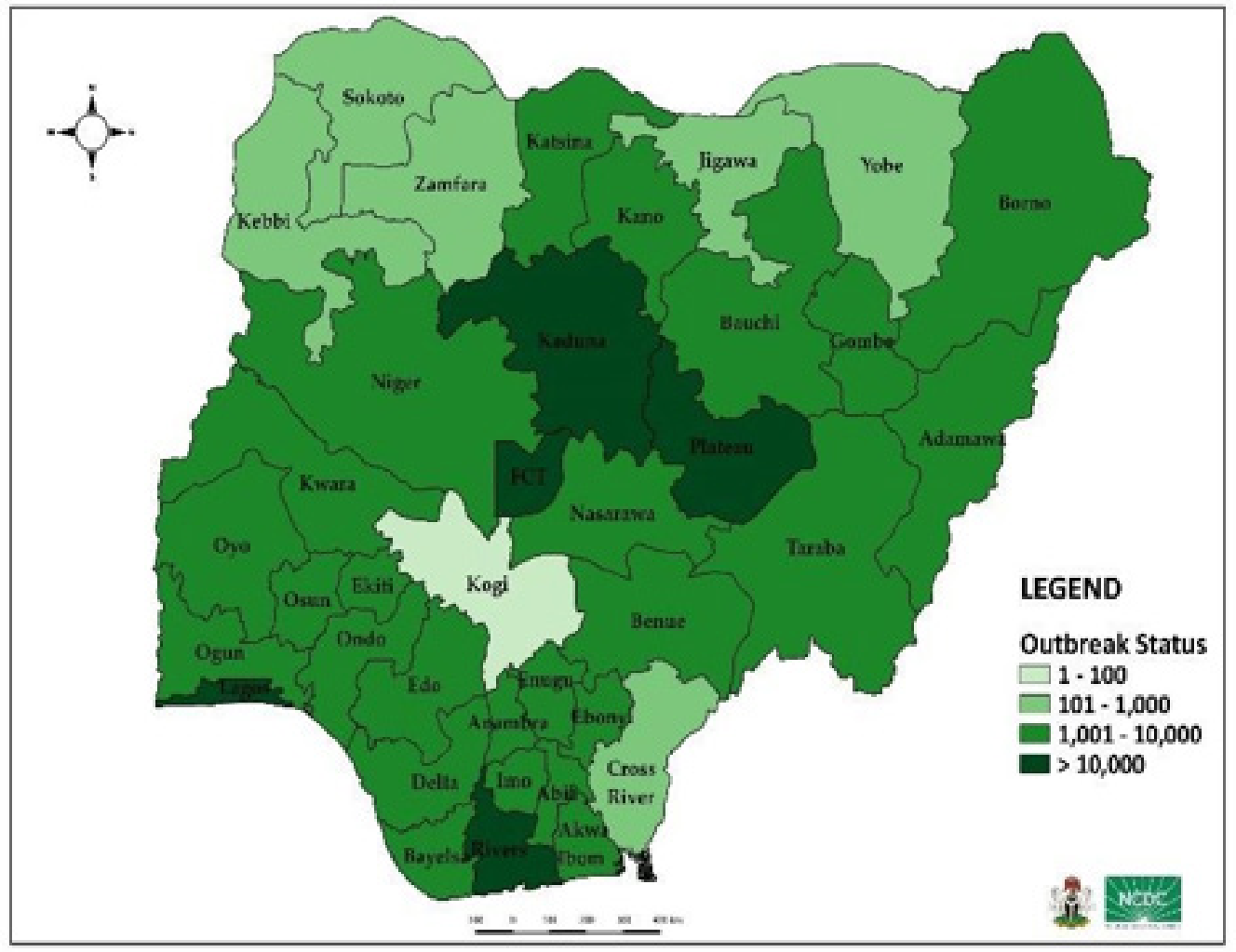

Figure 1: Distribution of confirmed COVID-19 cases by states in Nigeria February 2020 to December 2021 [9].

\section{Primary Health Care Structure and Service Delivery in Nigeria}

Primary Health Centres (PHCs) are the closest healthcare facilities to the majority of Nigerians [13]. PHCs has been at the center of the Nigerian health system for many years. It has been conceived as the key to providing basic health services to people with their full participation [12]. The system provides promotive, preventive, curative and rehabilitative services to the community. The basic health services provided by PHCs include but are not limited to immunization campaigns, maternal and child health services, infectious diseases surveillance and control, and resolution of uncomplicated health conditions [4]. The objectives of the PHC system includes establishing a health care system best adapted to the local conditions and level of health technology [13].

Nigeria is one of the signatories to the Alma-Ata declaration of PHC in 1978. But it is interesting to note that prior to the 1978 Alma-Ata declaration, the country had set the ball rolling with the implementation of the Basic Health Services Scheme (1975-1980), which was Nigeria's first serious attempt at the implementation of PHC [14]. In 1988, the National health policy of Nigeria was launched and is seen as a collective will of the government and people of Nigeria to provide a comprehensive health care system that is based on PHC. 
The national health policy, therefore, describes the goals, structure, strategies and policy direction of the health care delivery system in Nigeria [14].

In 1992, PHC implementation started with the commencement of PHC programs in the Local Government Areas (LGAs) [14]. PHC is provided by local government authority through health centers and health posts and they are staffed by nurses, midwives, community health officers, health technicians, community health extension workers and by physicians (doctors) especially in the southern part of the country [14]. Nigeria was among the first few countries in the developing world to have systematically decentralized the delivery of basic health services through local government administration [1416].

In order to ensure the sustainability of PHC in Nigeria, the Federal Government by decree number 29 of 1992, set up the National Primary Health Care Development Agency. This body was charged with the responsibility to mobilize support nationally and internationally for PHC program implementation [17-19, 14].

There are 774 Local Government Areas (LGA) in Nigeria with various health facilities operating under the hinges of primary health care (PHC). Evidence suggests that Nigeria has over 30,000 PHC facilities across the 774 LGAs in the 36 states and the Federal Capital Territory (FCT) [20]. The LGA coordinates the activities of the PHC system providing manpower, funds, logistics. The Local Government is headed by elected Chairmen with council members. Supervisory councilors are also appointed to oversee various aspects of Local Government activities including Health and Social Services [17]. The health department of the LGA is always headed by a Primary Health Care Coordinator or Medical Officer of Health.

\section{Key Roles of PHC System in COVID-19 Response in Nigeria}

PHC is essentially the cornerstone for the response to the COVID-19 outbreak globally because it is closest to the communities where cases reside, easily accessible and meets more than $80 \%$ of people's health needs especially in vulnerable communities [2,21]. It plays a significant role in gatekeeping and clinical responses in identifying and triaging potential COVID-19 cases, ensuring early diagnosis, helping vulnerable people cope with their anxiety, and reducing the hospital service demand [21]. Highlights of some key roles of the PHC system in sustaining the COVID-19 outbreak response in Nigeria are as follows

\section{Coordination of COVID-19 Outbreak Response through PHEOC}

Coordination of human, material and financial resources during an outbreak is key to prompt containment. During public health emergencies like the COVID-19 outbreak, enormous resources are required. However, the available resources are usually scarce especially if the outbreak is occurring simultaneously with other disease outbreaks as we have in Nigeria. Therefore, there is a need for proper coordination in order to effectively prioritize using available data for the proper and impactful allocation of available resources [11]. The LGA and PHC level is the operational base of every outbreak response because diseases occur at the community level and as such is most appropriate for proper coordination to be instituted at that level to drive every outbreak response especially using locally available resources which most times are very limited [22] Figure 2.

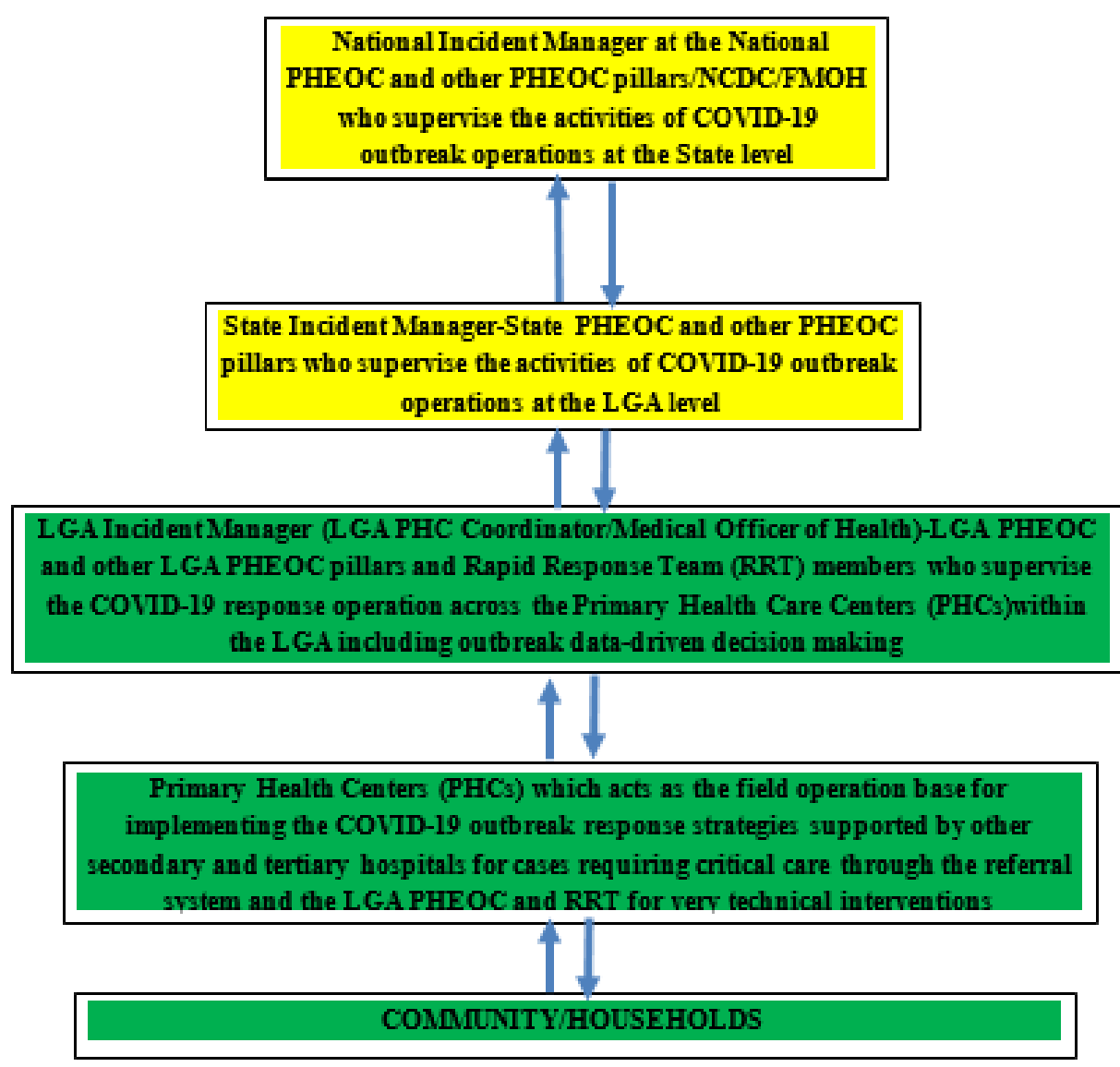

Figure 2: Framework for coordinating outbreak response with LGA and PHCs as the operational base [23]. 


\section{Enhancing Surveillance for Case Detection using COVID-19 Case Definition}

The PHC system could play a vital role in the COVID-19 pandemic response through early detection, community surveillance, and contact tracing. The most problematic areas to cover during epidemiologic surveillance are the rural communities due to logistical access problems like bad road networks [4]. This makes it more difficult to effectively respond to and control disease outbreaks in these communities [4]. However, the presence of PHCs can serve as a focal point for monitoring and responding to suspected cases, screening patients for likely symptoms, and tracing contacts of the confirmed case especially in rural inaccessible settings [4].

Also, the PHC system in Nigeria forms a greater proportion of the surveillance network in the country and as such can be used as a tool for strengthening community-based surveillance for COVID-19 case detection [24]. PHC cadre officers conduct regular follow-up home visits to households in communities under their jurisdiction and in the process could carry out active case searches for suspected cases of COVID-19 using case definition for prompt case detection [23].

Contact tracing for confirmed cases can best be implemented using a structure like the PHC framework that is closest to the cases and that rides on community participation [5]. A sustainable surveillance structure for COVID-19 contact tracing is the PHC system which encourages community participation through trust especially in a country like Nigeria where there is so much mistrust by the general public in the health system $[25,5]$.

\section{Support Scaling up of Access to Community Testing for COVID-19 Case Identification}

In Nigeria, one of the key challenges with the COVID-19 response is access to testing especially in hard-to-reach communities as there are limited molecular laboratories for COVID-19 testing with almost all located in the urban cities hampering access to testing for people in the rural communities except for the critically ill who will have to compulsorily transport themselves to the cities to access testing [5].

Though molecular PCR testing requires specialized skills and training which most PHC cadre staff might not have, however with the approval of COVID-19 Ag rapid testing kit for COVID-19 testing by WHO globally and the deployment by NCDC in Nigeria increased testing can be achieved through the PHC system framework. COVID-19 Ag$\mathrm{RDT}$ testing requires less specialized training and skills to perform which all PHC cadre staff under the strict supervision of the LGA primary health care coordinator (PHCC) have the capacity to perform. This will address the COVID-19 testing gaps between the residence of urban versus rural settlements presently observed in Nigeria

\section{Effective Home-Base Care and Management for Mild COVID-19 Confirmed Cases}

According to the federal ministry of health $(\mathrm{FMoH})$ of Nigeria guideline for home-based care for mild COVID-19 cases, all mild cases which are either pre-symptomatic or asymptomatic could be managed at home [26]. Previous studies in Nigeria have shown that more than $70 \%$ of COVID-19 cases are either asymptomatic or pre-symptomatic $[10,27,25]$. With the overwhelming proportion of confirmed cases on home-based care management especially as the country enters the fourth wave of COVID-19 outbreak, there is an urgent need to decentralize the case management approach using the PHC structure [26].

PHC cadre staff can play vital roles in daily monitoring and physical visits of mild COVID-19 cases at home for clinical progression and make urgent referral to a designated treatment center should these mild cases develop severe symptoms in the course of the homebased care monitoring exercise [26]. PHCs are mostly located in communities and are the closest health care system to mild COVID-19 confirmed cases. With the majority of the mild COVID-19 cases living in these areas and strong indications of community transmission of the disease, PHCs should serve as an important link in the management of the COVID-19 [28].

\section{Support Risk Communication and Social Mobilization for Increased Community Testing for COVID-19, Vaccination, and Compliance to Other Non-Pharmaceutical Preventive Measures}

Risk communication is the critical element in the fight against the COVID-19 pandemic. It is one of the core strategies in the COVID-19 outbreak response in Nigeria. However, operating a generalized risk communication and social mobilization approach despite the ethnic, socio-cultural and religious peculiarities of each region and community has made several risk communication efforts less impactful [25].

There are often misconceptions and misinformation about infectious disease outbreaks when they occur in Nigeria because of the multiethnic, socio-cultural and religious beliefs of her people. COVID-19 outbreak in Nigeria is no exception to this trend and this has hindered the implementation of key preventive measures [25].

Healthcare workers at PHCs within the communities could help dispel this by carrying out house-to-house visits to sensitize people about COVID-19 and emphasize preventive measures [28]. During the visits, they can carry out activities like teaching proper handwashing techniques and how to maintain social distancing, mobilizing people for COVID-19 testing and vaccination exercise [28]. And as respected members of their communities, people are more likely to listen to their advice and adhere to their instructions [28].

\section{Vaccination Campaigns for COVID-19}

PHC workers and infrastructure will play a central role in effective, equitable vaccination by increasing access to vaccines for vulnerable communities and providing communities with critical, accurate information about the safety and efficacy of vaccines to build patient confidence [1].

\section{Provision of Other Essential Health Services}

Evidence since the start of the COVID-19 pandemic has shown that the role of primary care during a public health emergency lean primarily to maintaining essential health services and secondly to responding to the outbreak [21]. During the pandemic, PHCs should be encouraged to continue providing services like immunization services, reproductive and adolescent health services, nutritional, antenatal, the elderly health care services, mental health and psychosocial support services although these services should be provided in ways that ensure physical distancing. Through that, community members would have more confidence in the primary health care system, and they will be encouraged to present to health facilities to seek healthcare [21].

\section{Conclusion}

Being the closest health care providers to people in communities and the first point of care in most occasions of health needs, $\mathrm{PHC}$ cadre staff can be trained to maintain a high index of suspicion while attending to patients. As a result, they will be able to detect suspicious cases early enough as they present to the facility, and prevent community spread. Also, Disease Surveillance and Notification Officers (DSNOs) at the local government level could be alerted early enough, and the suspected 
individual could be isolated and tested. In the event the test results are positive, the health workers could assist in conducting contact tracing in the communities and also home-based care management for mild cases and make an instant referral for severe cases, as they are familiar with the people within the host communities.

\section{Acknowledgments}

The author wish to appreciate all healthcare workers especially PHC staff who have been tirelessly involved in the fight against COVID-19 in Nigeria.

\section{References}

1. Primary Health Care Performance Initiative (PHCPI) (2021) Primary Health Care and COVID-19.

2. Akinwumi AF, Esimai OA, Fajobi O, Idowu A, Esan OT, et al. (2021) Knowledge of primary healthcare workers regarding the prevention and control of non-communicable diseases in Osun State, Nigeria: A ruralurban comparison. Afr J Prm Health Care Fam Med 13(1): e1-e8.

3. The Lancet (2018) The Astana Declaration: The future of primary health care? 392(10156): 1369.

4. Effiong FB, Elebesunu EE, Babatunde AO (2021) Primary Healthcare in Nigeria Amidst Disease Outbreaks: The Need for Improvements. Mod Care J 18(1): e113195.

5. Ajisegiri WS, Odusanya OO, Joshi R (2020) COVID-19 Outbreak Situation in Nigeria and the Need for Effective Engagement of Community Health Workers for Epidemic Response. Global Biosecurity 2(1).

6. Kadiri-Eneh NP, Uzochukwu BS, Tobin-West C, Azuile EC (2018) An assessment of job satisfactory among primary health care workers in Rivers state, Nigeria. Niger J Med 27(3): 282-291.

7. Sampson IT (2014) Religion and the Nigerian State: Situating the de facto and de jure Frontiers of State-Religion Relations and its Implications for National Security. Oxford Journal of Law and Religion 3(2): 311-339.

8. National Population Commission (NPC) [Nigeria] and ICF International (2014) Nigeria Demographic and Health Survey 2013. Abuja, Nigeria, and Rockville, Maryland, USA: NPC and ICF International. 1-2.

9. NCDC (2021) COVID-19 situation report, situation report 150.

10. Isere EE, Adejugbagbe AM, Fagbemi AT, Fagbemi S, Famokun AG, et al. (2021) Outcome of Epidemiological Investigation of COVID-19 Outbreak in a Southwest State of Nigeria, March to August 2020. Open Journal of Epidemiology 11(2): 163-177.

11. Oyebanji O, Ibrahim Abba F, Akande OW, Aniaku EC, Abubakar A, et al. (2021) Building local capacity for emergency coordination: establishment of subnational Public Health Emergency Operations Centres in Nigeria. BMJ Global Health 6(10): e007203.

12. Gyuse AN, Ayuk AE, Okeke MC (2018) Facilitators and barriers to effective primary health care in Nigeria. Afr J Prm Health Care Fam Med 10(1): e1-e3.

13. Metiboba S (2009) Primary Health Care Services for effective Health Care Development in Nigeria: a study of selected rural communities. Journal of Research in National Development 7(2): 58-64.

14. Alenoghena I, Aigbiremolen AO, Abejegah C, Eboreime E (2014) Primary Health Care in Nigeria: Strategies and Constraints in Implementation. IJCR 3(3): 74-79

15. Cueto M (2005) The Promise of Primary Health Care. Bull World Health Organ 83(5): 322 .

16. Obionu CN (2007) Primary Health Care For Developing Countries. $2^{\text {nd }}$ ed. Enugu: Delta Publications Nigeria. pp. 183-284.

17. Federal Ministry of Health Nigeria (FMOHN) (2004) Revised National Health Policy. Abuja: Federal Ministry of Health. p. 7-8.

18. Raids M (2008) Nigeria still searching for the right formula. Bull. World Health Organ 86(9): 663-665.

19. Magawa R (2002) Primary Health Care Implementation: A brief review.

20. Kress DH, Su Y, Wang H (2016) Assessment of primary health care system performance in Nigeria: Using the primary health care performance indicator conceptual framework. Health Systems \& Reform 2(4): 302318.

21. World Health Organization. Regional Office for the Western

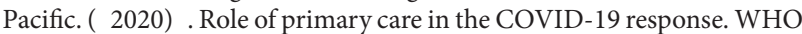
Regional Office for the Western Pacific.

22. Isere EE, Adedire EB, Ajayi IO (2019) Surveillance data: Generating credible statistics for policy making. In Selected topics in Medical Statistics. Adebowale SA, Akinyemi JO (Eds) University of Ibadan: ANDKOLAD Nigeria. pp. 508-530.

23. FMoH-NCDC. National Technical guideline for Integrated Disease Surveillance and Response. FMoH-NCDC $3^{\text {rd }}$ edition. pp. 188-189.

24. Isere EE, Fatiregun AA, Ajayi IO (2015) An overview of disease surveillance and notification system in Nigeria and the roles of clinicians in disease outbreak prevention and control. Niger Med J 56(3): 161-168.

25. Isere EE, Adejugbagbe AM, Oladoyin V, Abiona S, Omorogbe EN (2020) Pre-symptomatic and asymptomatic COVID-19 cases in Nigeria amidst prevailing socio-cultural beliefs and practices: Implication for COVID-19 transmission and way forward. Arch Prev Med 5(1): 39-42.

26. Federal Ministry of Health (2021) Interim Guidelines for Home Based Isolation and care of confirmed COVID-19 cases. FMoH. p. 13-23.

27. Bowale A, Abayomi A, Idris J, Omolabu S, Abdus-Salam I, et al. (2020). Clinical presentation, case management and outcomes for the first 32 COVID-19 patients in Nigeria. Pan African Medical Journal 35(Suppl 2): 24 .

28. Nkengasong J, Sirleaf EJ (2021) How health workers are leading Africa’s COVID-19 response. 